\title{
Classification of Opinion Mining Techniques
}

\author{
Nidhi Mishra \\ Assistant Professor, Department of Information \\ Technology, Buddha Institute of Technology \\ Gorakhpur, India
}

\author{
C.K.Jha, PhD. \\ Associate Professor, Department of AIM \& ACT, \\ Banasthali Vidyapith, Rajasthan India
}

\begin{abstract}
The important part to gather the information is always seems as what the people think. The growing availability of opinion rich resources like online review sites and blogs arises as people can easily seek out and understand the opinions of others. Users express their views and opinions regarding products and services. These opinions are subjective information which represents user's sentiments, feelings or appraisal related to the same. The concept of opinion is very broad. In this paper we focus on the Classification of opinion mining techniques that conveys user's opinion i.e. positive or negative at various levels. The precise method for predicting opinions enable us, to extract sentiments from the web and foretell online customer's preferences, which could prove valuable for marketing research. Much of the research work had been done on the processing of opinions or sentiments recently because opinions are so important that whenever we need to make a decision we want to know others' opinions. This opinion is not only important for a user but is also useful for an organization.
\end{abstract}

\section{Keywords}

Opinion Mining; Machine learning; Sentiments; Polarity; semantic.

\section{INTRODUCTION}

We Opinion mining is a type of natural language processing for tracking the attitudes, feelings or appraisal of the public about particular topic, product or services. Textual information in the entire world is of two types: facts and opinions. The facts are the objective expressions which describe the entities, events and properties whereas the opinion is the subjective expression which describes people's opinions, emotions and sentiments towards entities and their properties. The current search engine searches for facts because they assume the facts are true and can be expressed with keywords. But these search engines do not find the opinions because opinions or sentiments are very difficult to express by keywords and that is why there ranking strategy are not appropriate for opinion retrieval. Now, the web has significantly changed its way that people comments their views and opinion on any product and services. User can post their comments on any internet forums, review sites, blogs and discussion group which are commonly known as user generated content which contains the important information. This online word- of- mouth behavior represents new and considerable sources of information and their applications. These online comments are not limited to our friend circle but it is also expanded on a global or web scale. Today, if the user wants the views on a particular product he/she has no longer limited to ask their friends because they got the opinion for that product on the internet through various reviews or comments. In the same if the organization wants the opinion of their products and services they use these user generated contents from the web for the review or comments of their products and services. The introduction of Blog track in TREC 2006, a considerable work has been done in this field which comprises of opinion mining at sentence level, passage or document level and feature level. There are many challenges in opinion mining. The first challenges is that opinion word is considered to be positive in one condition may be considered negative in another condition. A second challenge is that people do not always state the opinions in a same way. The art Opinion Mining is to recognize the subjectivity and objectivity of a text and further classify the opinion orientation of subjective text. In short we say that Opinion Mining[19] is an automated extraction of subjective content from text and identifying the orientation such as positive or negative in that text. It aims to explore feelings of a person who write the text. It used Natural Language Processing and Machine Learning ethics to determine opinion in the text. The evaluation of opinion can be done in two ways:

- Direct opinion, gives positive or negative opinion about the object directly. For example, "The picture quality of this camera is poor" expresses a direct opinion.

- Comparison means to compare the object with some other similar objects. For example, "The picture quality of camera-y is better than that of Camera-x." expresses a comparison.

Spam filtering refers to detection and removal of fake opinions that mislead the users by giving unworthy positive or negative opinions to some objects in order to sponsor or spoil the objects reputations. It is also a research issue in healthy opinion mining. The applications of opinion mining are in (1) Argument mapping software helps organizing in a logical way these policy statements, by explicitating the logical links between them (2) Voting Advise Applications: help voters to understand which political party have closer positions with them. (3) Automated content analysis helps processing large amount of qualitative data. There are lots of Free and Open Source tools available for performing Natural Language Processing and Machine Learning tasks. Also the huge amount of user generated content, organized for opinion mining task is available on web. Following tools like GATE, NLTK, Apache Mahout, Weka, Rapidminer, KNIME, and Open NLP etc. can be used to develop your own opinion mining automated system. This paper is an extension to our work is which is carried out in my paper [28] titled- "An insight into task of opinion mining" accepted in Springer SPIT-2012 International Conference.

Rest of the paper is organized as follows Section 2 deals about literature review of classification of opinion mining techniques and opinion mining in compound sentence. Section 3 deals the discussion and comparison of various opinion mining techniques. In section 4 discuss about various 
opinion mining tools. Finally we conclude our discussion in Section 5.

\section{LITERATURE REVIEW AND TASK OF OPINION MINING}

All In order to give more imminent into the problem of opinion mining, in the following sections we discuss the domain overview and various types of opinion mining. The opinion mining is frequently associated with the topic information retrieval. The information retrieval algorithm works on factual data but the opinion mining works on subjective data. The task of opinion mining is to find the opinion of an object whether it is positive or negative and what features does it depict, and what features are appreciated, which are not etc. The notion of an opinion mining is given by $\mathrm{Hu}$ and Liu [2]. They put most impact on their work and said that the basic components of an opinion are:

- Opinion holder: it is the person that gives a specific opinion on an object.

- Object: it is entity on which an opinion is expressed by user.

- Opinion: it is a view, sentiment, or appraisal of an object done by user.

\subsection{Task of Opinion Mining at Document level}

Document level opinion mining is about classifying the overall opinion presented by the authors in the entire document as positive, negative or neutral about a certain object [3] [4]. The assumption is taken at document level is that each document focus on single object and contains opinion from a single opinion holder. Turney [27] present a work based on distance measure of adjectives found in whole document with known polarity i.e. excellent or poor. The author presents a three step algorithm i.e. in the first step; the adjectives are extracted along with a word that provides appropriate information. Second step, the semantic orientation is captured by measuring the distance from words of known polarity. Third step, the algorithm counts the average semantic orientation for all word pairs and classifies a review as recommended or not. In contrast, Pang et al. [5] present a work based on classic topic classification techniques. The proposed approach aims to test whether a selected group of machine learning algorithms can produce good result when opinion mining is perceived as document level, associated with two topics: positive and negative. He present the results using nave bayes, maximum entropy and support vector machine algorithms and shown the good results as comparable to other ranging from 71 to $85 \%$ depending on the method and test data sets. Apart from the document-level opinion mining, the next sub-section discusses the classification at the sentence-level, which classify each sentence as a subjective or objective sentence and determine the positive or negative opinion.
2.2 Task of opinion mining at Sentence level

The sentence level opinion mining is associated with two tasks [6] [7] [8]. First one is to identify whether the given sentence is subjective (opinionated) or objective. The second one is to find opinion of an opinionated sentence as positive, negative or neutral. The assumption is taken at sentence level is that a sentence contain only one opinion for e.g., "The picture quality of this camera is good." However, it is not true in many cases like if we consider compound sentence for e.g., "The picture quality of this camera is amazing and so is the battery life, but the viewfinder is too small for such a great camera", expresses both positive and negative opinions and we say it is a mixed opinion. For "picture quality" and "battery life", the sentence is positive, but for "viewfinder", it is negative. It is also positive for the camera as a whole. Riloff and Wiebe [11] use a method called bootstrap approach to identify the subjective sentences and achieve the result around 90\% accuracy during their tests. In contrast, Yu and Hatzivassiloglou [13] talk about sentence classification (subjective/objective) and orientation (positive/negative/neutral). For the sentence classification, author's present three different algorithms: (1) sentence similarity detection, (2) naïve Bayens classification and (3) multiple naïve Bayens classification. For opinion orientation authors use a technique similar to the one used by Turney [27] for document level. Wilson et al. [12] pointed out that not only a single sentence may contain multiple opinions, but they also have both subjective and factual clauses. It is useful to pinpoint such clauses. It is also important to identify the strength of opinions. Like the document-level opinion mining, the sentence-level opinion mining does not consider about object features that have been commented in a sentence. For this the feature level opinion mining is discuss in the next subsection.

\subsection{Task of Opinion mining at Feature level}

The task of opinion mining at feature level is to extracting the features of the commented object and after that determine the opinion of the object i.e. positive or negative and then group the feature synonyms and produce the summary report. Liu [16] used supervised pattern learning method to extract the object features for identification of opinion orientation. To identify the orientation of opinion he used lexicon based approach. This approach basically uses opinion words and phrase in a sentence to determine the opinion. The working of lexicon based approach [18] is described in following steps.

- Identification of opinion words

- Role of Negation words

- But-clauses 
Table 1. Presents insight into opinion mining at different levels[28]

\begin{tabular}{|c|c|c|}
\hline $\begin{array}{l}\text { Classification } \\
\text { of Opinion } \\
\text { mining at } \\
\text { different } \\
\text { levels }\end{array}$ & $\begin{array}{l}\text { Assumptions made at } \\
\text { different levels }\end{array}$ & $\begin{array}{l}\text { Tasks associated } \\
\text { with different } \\
\text { levels }\end{array}$ \\
\hline $\begin{array}{ll}\text { 1. } & \text { Opinion } \\
\text { Mining at } \\
\text { Sentence } \\
\text { level. }\end{array}$ & $\begin{array}{l}\text { 1. A sentence } \\
\text { contains only one } \\
\text { opinion posted by } \\
\text { single opinion } \\
\text { holder; this could } \\
\text { not be true in } \\
\text { many cases e.g. } \\
\text { there could be } \\
\text { multiple opinions } \\
\text { in compound and } \\
\text { complex } \\
\text { sentences. } \\
\text { Secondly the } \\
\text { sentence } \\
\text { boundary is } \\
\text { defined in the } \\
\text { given document }\end{array}$ & $\begin{array}{l}\text { Task 1: } \\
\text { identifying the } \\
\text { given sentence as } \\
\text { subjective or } \\
\text { opinionated } \\
\text { Classes: } \\
\text { objective and } \\
\text { subjective } \\
\text { (opinionated) } \\
\text { Task 2: opinion } \\
\text { classification of } \\
\text { the given } \\
\text { sentence. } \\
\text { Classes: positive, } \\
\text { negative and } \\
\text { neutral. }\end{array}$ \\
\hline $\begin{array}{ll}\text { 2. } & \text { Opinion } \\
& \text { Mining at } \\
\text { Document } \\
\text { level. }\end{array}$ & $\begin{array}{ll}\text { 1. } & \begin{array}{l}\text { Each document } \\
\text { focuses on a } \\
\text { single object and } \\
\text { contains opinion } \\
\text { posted by a single }\end{array} \\
\text { opinion holder. } \\
\text { 2. } & \text { Not applicable } \\
\text { for blog and } \\
\text { forum post as } \\
\text { there could be } \\
\text { multiple opinions } \\
\text { on multiple } \\
\text { objects in such } \\
\text { sources. }\end{array}$ & $\begin{array}{l}\text { Task 1: opinion } \\
\text { classification of } \\
\text { reviews } \\
\text { Classes: positive, } \\
\text { negative, and } \\
\text { neutral }\end{array}$ \\
\hline $\begin{array}{ll}\text { 3. } & \text { Opinion } \\
\text { Mining at } \\
\text { Feature } \\
\text { level. }\end{array}$ & $\begin{array}{l}\text { 1. The data source } \\
\text { focuses on } \\
\text { features of a } \\
\text { single object } \\
\text { posted by single } \\
\text { opinion holder. } \\
\text { 2. Not applicable } \\
\text { for blog and } \\
\text { forum post as } \\
\text { there could be } \\
\text { multiple opinions } \\
\text { on multiple } \\
\text { objects in such } \\
\text { sources. }\end{array}$ & $\begin{array}{l}\text { Task 1: Identify } \\
\text { and extract object } \\
\text { features that have } \\
\text { been commented } \\
\text { on by an opinion } \\
\text { holder (e.g., a } \\
\text { reviewer). } \\
\text { Task 2: } \\
\text { Determine } \\
\text { whether the } \\
\text { opinions on the } \\
\text { features are } \\
\text { positive, negative } \\
\text { or neutral. } \\
\text { Task 3: Group } \\
\text { feature } \\
\text { synonyms. } \\
\text { Produce a } \\
\text { feature-based } \\
\text { opinion summary } \\
\text { of multiple } \\
\text { reviews. }\end{array}$ \\
\hline
\end{tabular}

In contrast, $\mathrm{Hu}$ and Liu do customer review analysis [26] through opinion mining based on feature frequency, in which the most frequent features is accepted by processing many reviews that are taken during summary generation. In opposite to $\mathrm{Hu}$ and Liu, Popescu and Etzioni [20], improved the frequency based approach by introducing the part-of relationship and remove the frequent occurring of noun phrases that may not be features.

\subsection{Opinion Mining in Compound sentence}

In this sub-section the following methodology we use to determine the opinion in compound sentence of a movie review domain:

\subsubsection{Sentence classification}

In the sentence classification we go to individual compound sentences to determine whether a sentence is subjective or express an opinion and if so, whether the opinion is positive or negative (called sentence-level sentiment classification). For example, 'Desi Boyz' - highly entertaining comedy gives the positive opinion and 'Damadamm' clearly has no Dum gives the negative opinion.

The following activities are done within sentence classification:

\subsubsection{Splitting of the document into sentences}

Given a document about the movie reviews, the document is segmented into individual sentences by the help of sentence delimiter. Here problem is that most of the reviews are found on movie forums or blog sites where normal users post their opinions in their informal language which do not follow strict grammatical rules and punctuations. The identification of full stop in the sentence does not mark the end of sentence sometimes. Such as date 12.1.2012, movie short forms T.M.K expressed in example 1, hence we have to use rule based pattern matching to identify sentence boundary. Second problem is that people generally use internet slang words like OMG, cuteeeee etc. e.g. actress is cuteeee. Here there is not such word in dictionary like cuteeee but it refers to cute. We will do $\mathrm{N}$ gram matching of such words with pre compiled dictionary of movie related words. The output of this splitting document into sentences yields following:

- $\quad$ M Gud reviews about film released on 12.1.2012.

- He says, "The film T.M.K's story is filled with a great plot, the actors are first grade, and actress is cuteeee".

- The supporting cast is good as well, but, movie can't hold up.

\subsubsection{Determining whether the sentence is opinionated}

We will use boot strap approach proposed by Riloff and Wiebe [11] for the task of subjective sentences identification. It will use a high precision (and low recall) classifiers to extract a number of subjective sentences collected from various movie review sites. From this subjective sentence a set of patterns will be learned. The learned patterns will be used to extract more subjective and objective sentence. The subjective classifier will look for the presence of words from the pre compiled list, while the objective classifier tries to locate sentences without those words. In the example 1 all sentences except " $M$ Gud reviews about film released on 12.1.2012" are opinionated. 


\subsubsection{Determining whether the opinionated sentence is compound sentence}

A compound sentence is a sentence that contains two or more complete ideas (called clauses) that are related. These two or more clauses are usually connected in a compound sentence by a conjunction. The coordinating conjunctions are "and", "but", "for", "or", "nor", "yet", or "so". We will use plain pattern matching to find out the presence of coordinating conjunctions. If they are present in the given sentence then it will be identified as compound sentence. Output of this will be set of compound sentences

- He says, "The film T.M.K's story is filled with a great plot, the actors are first grade, and actress is cuteeee".

The supporting cast is good as well, but, movie can't hold up

The compound sentence is a sentence which expresses more than one opinion of an object. For example, the sentence, "The supporting cast is good as well, but, movie can't hold up.", expresses both positive and negative opinions (one may say that it has a mixed opinion). For "supporting cast", the sentence is positive, but for "overall", it is negative. It is also positive for the movie as a whole.

\subsubsection{Opinion Mining in Compound sentence}

In this section we focus on opinion expressions in the compound sentence of a movie review that gives the opinion on the individual feature of the movie and the opinion of the movie as whole i.e. positive or negative sentiments. Apart from this we also determine the sentiment score towards various features of a movie, such as cast, director, story and music. Sentiment scores are used to classify the sentiment polarity (i.e. Positive, negative or neutral) of clauses or sentences. The linguist approach makes use of both a domain-specific lexicon (specify the noun related terms like actor, director etc.) and a generic opinion lexicon(specify the property of movie related terms), derived from SentiWordNet[9], to assign a prior sentiment score to each word in a sentence. For example,

- He says, "The film T.M.K's story is filled with a great plot, the actors are first grade, and actress is cuteeee.

- The supporting cast is good as well, but, movie can't hold up.

The compound sentences ( 1 and 2 ) are divided into "The film T.M.K's story is filled with a great plot" "the actors are first grade" "actress is cute"; "The supporting cast is good as well" and "movie can't hold up". After dividing the compound sentences into separate sentences or clauses, a sentiment score toward each movie features (e.g. Story, actors, actress, supporting cast or the film features) is calculated. (For example like the sentiment score is from 0 to 5 here 0 indicates the most negative opinion ,5 indicate the most positive opinion and between 0 to 5 we have to make the rules for positive opinion and negative opinion.) After calculating the sentiment score for each clause or sentence, the sentiment score for each review features and the overall sentiment score for the whole sentence is calculated. The following tools we use like: Sentence Delimiter [15], Part of Speech tagger [14], Named Entity recognition [14], Sentiword Net [9] for determine the opinion in compound sentence.

\section{DISCUSSION AND COMPARISON}

The performance of opinion mining in determining the orientations or polarity is evaluated by calculating various metrics like precision, recall and F-measure. The overview of the work done in the task of opinion mining and its orientations is discussed and compare in the Table II [17]. It is seen from the Table II, as the data source is concerned, a huge amount of work has been done on movie and product reviews to determine the opinion orientations. The Internet Movie Database is used for movie reviews and product reviews are taken from Amazon.com. Movie review is a more challenging application than many other types of review mining. The challenges of movie review based on the factual information which is always mixed with real-life data and mocking words are used in writing movie reviews. The Product review domain considerably differs from movie review domain because of the following reasons. One reason is that there are feature specific comments in product reviews because people may like some features and dislike others. Thus reviews consists opinions orientations in the text, which is a difficult one to classifying opinion orientation of review as positive or negative. Following feature specific reviews occur less often in movie reviews. Second reason is that there are a lot of comparative sentences in product reviews and people discuss about other products in reviews.

\section{TOOLS USED IN OPINION MINING}

The tools which are used to track the opinion or polarity from the user generated contents are:

- $\quad$ Review Seer tool [17] - This tool is used to automates the work done by aggregation sites. The Naive Bayes classifier approach is used to collect positive and negative opinions for assigning a score to the extracted feature terms.

- Web Fountain [25]- It uses the beginning definite Base Noun Phrase (bBNP) heuristic approach for extracting the product features.

- Red Opal [24] -It is a tool that enables the users to determine the opinion orientations of products based on their features. It assign the scores to each product based on features extracted from the customer reviews'

- Opinion observer[17]-This is an opinion mining system for analyzing and comparing opinions on the Internet using user generated contents. This system shows the results in a graph format showing opinion of the product feature by feature.

Along with these automated tools, there are various online tools like Twitrratr, Twendz, Social mention, and Sentimetrics are available to track the opinions in the web. 
Table 2. Summary based on literature survey of opinion mining[17]

\begin{tabular}{|c|c|c|c|c|c|c|c|c|}
\hline 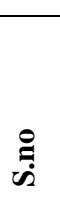 & 莺 & 象苛 & 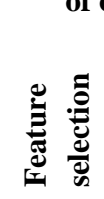 & 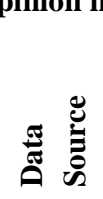 & ه & 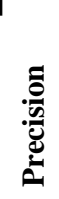 & $\begin{array}{l}\overline{\bar{\varpi}} \\
\approx \\
\approx\end{array}$ & $\Phi$ \\
\hline- & 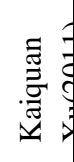 & 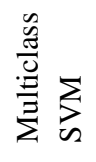 & 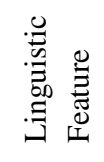 & 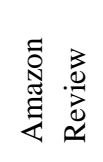 & $\frac{\circ}{6}$ & $\frac{\stackrel{\circ}{\sigma}}{\sigma}$ & \begin{tabular}{l}
$\stackrel{\circ}{+}$ \\
\multirow{\sigma}{\alpha}{}
\end{tabular} & 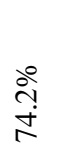 \\
\hline$\alpha$ & $\bar{z}$ & 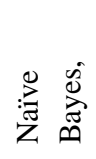 & 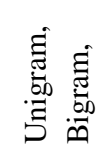 & $\frac{0}{3} \frac{0}{3}$ & $\begin{array}{l}\stackrel{0}{ } \\
\infty \\
\infty \\
\infty \\
0 \\
\ddot{z}\end{array}$ & , & , & , \\
\hline$m$ & 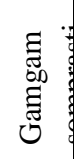 & 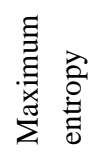 & 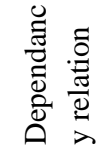 & 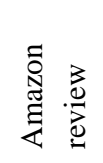 & , & $\begin{array}{l}\stackrel{b}{0} \\
\stackrel{i}{i}\end{array}$ & $\begin{array}{l}\stackrel{D^{\circ}}{2} \\
\stackrel{\infty}{r}\end{array}$ & $\begin{array}{l}\stackrel{\circ}{+} \\
\stackrel{2}{r}\end{array}$ \\
\hline+ & 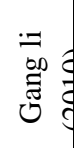 & 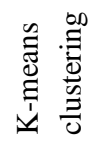 & 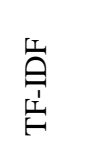 & 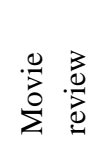 & $\stackrel{\circ}{\infty}$ & , & , & \\
\hline in & $\frac{0}{\frac{2}{5}}$ & 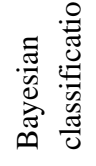 & 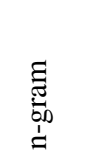 & $\begin{array}{l}60 \\
\frac{6}{0} \\
\frac{0}{0}\end{array}$ & $\frac{\infty}{\infty} \frac{\stackrel{\circ}{c}}{\circ}$ & , & , & ' \\
\hline 0 & 莺 & 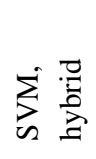 & 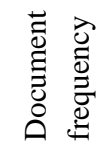 & $\begin{array}{l}\frac{0}{2} \\
\sum_{2}^{0} \\
\sum_{0}^{0}\end{array}$ & $\stackrel{\circ}{\infty}$ & , & , & , \\
\hline$r$ & 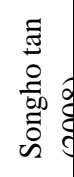 & 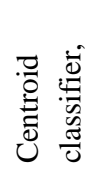 & 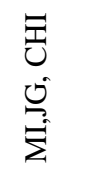 & 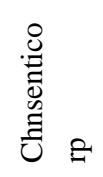 & $\begin{array}{l}\sum_{2} \\
2 \\
80 \\
8\end{array}$ & , & , & , \\
\hline$\infty$ & $\begin{array}{l}\frac{0}{0} \\
\overline{0} \\
0 \\
0\end{array}$ & 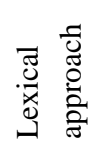 & 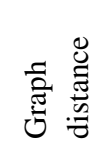 & $\begin{array}{l}0 \\
00 \\
0 \\
0 \\
00 \\
0 \\
0\end{array}$ & 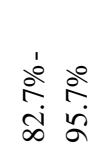 & , & , & \\
\hline$a$ & 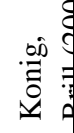 & $\stackrel{\overrightarrow{0}}{\vec{D}}$ & 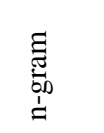 & 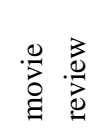 & $\frac{\circ}{a}$ & , & , & , \\
\hline 으 & है & 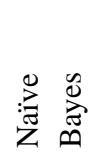 & 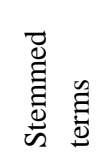 & 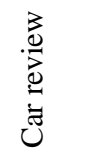 & ஷ̊రి & , & , & ' \\
\hline$=$ & 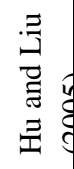 & 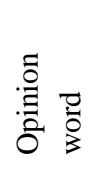 & 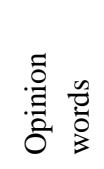 & 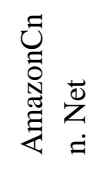 & 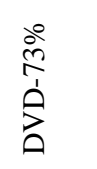 & , & , & , \\
\hline$\simeq$ & $\begin{array}{l}\vec{\sigma} \\
\bar{\sigma} \\
\text { on } \\
\bar{\Xi} \\
\tilde{\sigma}\end{array}$ & 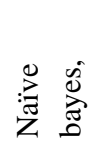 & 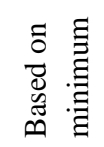 & $\begin{array}{l}\frac{3}{3} \\
\frac{0}{2} \\
\sum_{0}^{2}\end{array}$ & $\begin{array}{l}\stackrel{\circ}{+} \\
\dot{\infty} \\
\dot{\infty}\end{array}$ & , & , & \\
\hline
\end{tabular}

\section{CONCLUSIONS}

This paper presents the classification of opinion mining techniques. Opinion mining aims at recognizing, classifying and determining opinion orientations of the opinionated text. In this paper we first presented a theoretical model of opinion mining, which discuss the problem and supply a common outline in different research directions. It then discussed in section 2 the most usually topic task of opinion mining techniques at various level, which determines whether a document or sentence is carries a positive or negative opinion. We also discuss the feature level opinion mining. We observe that the opinion mining in compound sentence is also very challenging task which we already discuss in section 2 . In section 3 we discuss about comparison of various opinion mining techniques. Last but not the least we discuss about the opinion spams in text. Detecting fake opinions is a research issue. Some of the opinions are time sensitive hence identifying latest opinions on the subject is another research issue. In section 4 we discuss about various opinion mining tools used to track the opinion in the web. Finally, we conclude the paper by proverb that all the tasks of opinion mining and detecting the opinion orientation are very challenging. Our understanding and knowledge of the problem and its solution are still limited.

In future, more work is needed on further improving the performance measures. Opinion Mining can be applied for many new applications. The techniques and algorithms used for opinion mining are very fast, and many of the study remain unsolved. The main challenging aspects exist in the use of opinion mining classifications, dealing with negation expressions; produce a summary of opinions based on product features, complexity of sentence/document, handling of hidden product features, etc. Many future research works could be committed to these challenges.

\section{REFERENCES}

[1] A. Berger, S. Della Pietra, and V.J. Della Pietra 1996. A maximum entropy approach to natural language processing , Computational Linguistics.

[2] B. Liu 2007. Web Data Mining, Exploring Hyperlinks, Contents and Usage data.

[3] B. Liu 2011. Opinion Mining and Sentiment Analysis, AAAI, San Francisco, USA.

[4] B.Liu 2010. Opinion Mining and Sentiment Analysis: NLP Meets Social Sciences", STSC, Hawaii.

[5] B. Liu 2008. Opinion Mining and Summarization, World Wide Web Conference, Beijing, China.

[6] B. Liu. 2010. Sentiment Analysis: A Multifaceted Problem., Invited paper, IEEE Intelligent Systems.

[7] B. Liu. 2010 Sentiment Analysis and Subjectivity Second Edition, The Handbook of Natural Language Processing.

[8] B. Pang, L. Lee, and S. Vaithyanathan, 2002. Thumbs up? Sentiment classification using machine learning techniques," Proceedings of the Conference on Empirical Methods in Natural Language Processing (EMNLP), pp. 79-86.

[9] C. Cardie, J. Wiebe, T. Wilson, and D. Litman, 2003. Combining low-level and summary representations of opinions for multi- perspective question answering, 
Proceedings of the AAAI Spring Symposium on New Directions in Question Answering, pp. 20-27.

[10] ComScore/the Kelsey group 2007. Online consumergenerated reviews have significant impact On offline purchase behavior, Press Release. http://www.comscore.com/press/release.asp?press=1928

[11] E. Riloff, and J. Wiebe, 2003. Learning Extraction Patterns for Subjective Expressions, Proceedings of the Conference on Empirical Methods in Natural Language Processing (EMNLP), Japan, Sapporo.

[12] T.Wilson,., J. Wiebe,., R. Hwa,. 2004. Just how mad are you? Finding strong and weak opinion clauses. In: the Association for the Advancement of Artificial Intelligence, pp. 761--769.

[13] H. Yu, and V. Hatzivassiloglou, 2003. Towards Answering Opinion Questions: Separating Facts from Opinions and Identifying the Polarity of Opinion Sentences, Proceedings of the Conference on Empirical Methods in Natural Language Processing (EMNLP), Japan, Sapporo.

[14] W. Jin, H. Hay Ho, and R. Srihari, 2009. Opinion Miner: A Novel Machine Learning System for Web Opinion Mining and Extraction. Proceeding of International conference on Knowledge Discovery and Data Mining Paris, France.

[15] L. Dey, S.K. Mirajul Haque, 2009. Studying the effects of noisy text on text mining applications. Proceedings of the Third Workshop on Analytics for Noisy Unstructured Text Data, Barcelona, Spain.

[16] B. Liu, and J. Cheng, 2005. Opinion observer: Analyzing and comparing opinions on the web, Proceedings of WWW.

[17] G.Vinodhini and RM. Chandrasekaran 2012. Sentiment analysis and Opinion Mining: A survey International Journal of advanced Research in Computer Science and Software Engineering vol. 2 Issue 6.

[18] X. Ding, B. Liu, and P. S. Yu, 2008. A holistic lexiconbased approach to opinion mining, Proceedings of the Conference on Web Search and Web Data Mining (WSDM)
[19] G. Jaganadh 2012. Opinion mining and Sentiment analysis CSI Communication.

[20] A.M..Popescu,, O. Etzioni, 2005. Extracting Product Features and Opinions from Reviews, In Proc. Conf. Human Language Technology and Empirical Methods in Natural Language Processing, Vancouver, British Columbia, pp. 339-346.

[21] ZHU Jian, XU Chen, and WANG Han-shi, 2010. Sentiment classification using the theory of ANNs, The Journal of China Universities of Posts and Telecommunications, 17(Suppl.): pp. 58-62.

[22] J. Martin 2005. Blogging for dollars. Fortune Small Business, 15(10), pp. 88-92.

[23] J. Wiebe, E. Breck, C. Buckley, C. Cardie, P. Davis, B. Fraser, D. Litman, D. Pierce, E. Riloff, T. Wilson, D. Day, and M. Maybury, 2003. Recognizing and organizing opinions expressed in the world press in Proceedings of the AAAI Spring Symposium on New Directions in Question Answering.

[24] Christopher Scaffidi, Kevin Bierhoff, Eric Chang, Mikhael Felker, Herman Ng and Chun Jin 2007. Red Opal: product-feature scoring from reviews, Proceedings of 8th ACM Conference on Electronic Commerce, pp. 182-191, New York.

[25] Yi and Niblack 2005. Sentiment Mining in Web Fountain" ,Proceedings of 21st international Conference on Data Engineering, pp. 1073-1083, Washington DC.

[26] M. Hu and B. Liu 2004. Mining and summarizing customer reviews, Proceedings of the ACM SIGKDD Conference on Knowledge Discovery and Data Mining (KDD), pp. 168-177.

[27] P.Turney 2002. Thumbs Up or Thumbs Down? Semantic Orientation Applied to Unsupervised Classification of Reviews. In: Proceeding of Association for Computational Linguistics, pp. 417--424.

[28] N.Mishra and C.K.Jha 2012. An insight into task of opinion mining Second International Joint Conference on Advances in Signal Processing and Information Technology - SPIT. 